\title{
MONITORIZACIÓN DE LOS CAMBIOS DE USO DEL SUELO EN LA CABECERA DE CUENCA DE LA RIBERA SALADA MEDIANTE FOTOGRAFÍA AÉREA Y S.I.G. (EL SOLSONĖS, LLEIDA, ESPAÑA)
}

\author{
J. M. Ubalde, J. RIUS \& R. M. POCH ${ }^{*}$
}

Centre Tecnològic Forestal de Catalunya - Departament de Medi Ambient $i$ Ciències del Sòl, Universitat de Lleida. Av. Rovira Roure, 177. E-25198 Lleida.c. e.: rosa.poch@macs.udl.es

RESUMEN.- Durante estos últimos 50 años ha tenido lugar un abandono de las masías y de los cultivos tradicionales en los Prepirineos catalanes. En este trabajo se han estudiado los cambios de usos del suelo desde los años 50 hasta la actualidad en una cuenca de 6577 ha situada en la cabecera de la Ribera Salada (El Solsonès). Para ello se han utilizado fotografías aéreas del año 1957 y del año 1992. Como base cartográfica sirvieron los ortofotomapas del año 1993. El análisis de los cambios de usos según diferentes variables (pendiente y orientación) y segun diferentes enfoques (intensidad del cambio y uso final), se ha llevado a cabo mediante el programa de SIG ARC/INFO ${ }^{\otimes}$. Se han establecido tendencias en la evolución de los usos según la pendiente y la orientación. Los resultados obtenidos muestran que los campos de cultivo son los que han disminuido más en superficie, dando lugar a pastos. Por otro lado, el bosque denso ha aumentado considerablemente su extensión ( $845 \mathrm{ha}$ ).

ABSTRACT.- During the last 50 years an abandonment of the traditional crops and rural settlements has taken place in the catalan Prepyrenees. In this study the land use changes observed from the fifties to the present day have been monitored in a 6577 ha-watershed located in the headwaters of the Ribera Salada (El Solsonès). In order to do that aerial photographs from the years 1957 and 1993 have been compared, using 1993-ortophotomaps as cartographic base. The analysis of the land use changes according to different variables (aspect and slope) and different approaches (change intensity and final land use) has been performed using $A R C / I N F O^{\infty}$. Several trends have been established in the land use evolution according to aspect and slope. The results show that the crops are the land use with the maximum decrease in surface, which has taken place mostly on steep slopes, giving place to meadows. On the other side, the surface occupied by dense forests has considerably increased $(845 \mathrm{ha}$ ). 
PIRINEOS 153-154

RÉSUMÉ.- Pendant les derniers 50 années il y a eu un abandon de maisons rurales et de cultures traditionnelles dans les Prépyrénées catalans. Dans ce travail nous avons étudié les changements d'utilisation du sol depuis les années 50 jusqu'à présent dans un bassin versant de 6577 ha situé aux sources de la "Ribera Salada» (El Solsonès). La méthodologie choisie a été la comparaison de photographies aériennes des années 1957 et 1992, avec les orthophotomapes de l'année 1993 comme base cartographique. L'analyse des changements a été faite selon différentes variables (pente et orientation) et avec différentes approches (intensité et utilisation finale) avec le SIG ARC/INFO®. Cette méthodologie a permis l'établissement de tendances évolutives en fonction de la pente et de l'orientation des versants. Les résultats obtenus montrent que les cultures sont le type d'utilisation du sol qui a diminué le plus en surface occupée, principalement sur les pentes fortes, occupées au présent par des pâturages. D'autre part, la forêt dense a augmenté considérablement sa surface (845 ha).

Keywords: Land use, Prepyrenees, abandonment, GIS.

\section{Introducción}

En los últimos 50 años, tanto en los Prepirineos como en otras montañas españolas, ha habido un pronunciado proceso de despoblación. Anteriormente había grandes superficies cultivadas; a menudo en condiciones físicas muy adversas. En las montañas de Burgos el $45 \%$ de la superficie estaba cultivada, en el Valle de Jubera (Sistema Ibérico) el $35 \%$ y un $35.4 \%$ en las Alpujarras de Granada (ORTEGA VALCÁRCEL, 1974; GARCÍA-RUIZ et al., 1985; BOSQUE, 1968). Las dificultades del medio físico, la modernización de las explotaciones agrícolas, la falta de equipamientos y el aislamiento de las zonas de montaña, entre otros factores, han hecho que en las últimas décadas hubiera una descenso del área cultivada y, consecuentemente, un incremento del número de campos abandonados. Entre 1940 y 1975, alrededor del $40 \%$ de la tierra cultivada ha sido abandonada en la Cabrera leonesa, el $89 \%$ en las solanas de la Sierra de Ayllón y el $100 \%$ en muchos valles de Cameros (CABERO, 1980; BORDIU, 1985; GARCÍA-RUIZ et al., 1985). En los Pirineos Centrales este abandono ha sido de un $70 \%$ (LASANTA, 1988).

En la cuenca estudiada, igual que en todo el Pirineo, el abandono de tierras agrícolas también ha tenido lugar. Ello tiene importantes implicaciones medioambientales y socioeconómicas. Hay cambios en el funcionamiento hidrológico (DEBUSSCHE et al., 1987) y geomorfológico de las laderas y aparece una competencia entre la colonización vegetal y la instalación de ciertos procesos erosivos (GARCÍA-RUIZ, 1988). Según LASANTA (1989) el modelo de variación sigue dos fases: hasta la primera mitad del siglo se abandonan los campos menos fértiles, y sólo posteriormente se abandonan los menos meca- 


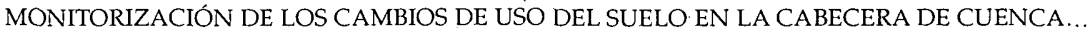

nizables o accesibles. Ello conduce a la intensificación del uso de las mejores áreas y la subutilización del resto (LASANTA \& RUIZ FLAÑO, 1990).

En este abandono influyen diversos factores físicos y socioeconómicos, que explican como se ha producido esta reducción de la superficie cultivada. Varios autores han relacionado los cambios con variables territoriales. GÓMEZ-SAL et al. (1993) muestran, tras un análisis estadístico de los modelos de variación en la Cordillera Cantábrica, un aumento de la diversidad y una regresión de las comunidades vegetales de pastos a matorrales. Las relaciones con otros parámetros indican que las primeras zonas en abandonarse son las de más pendiente, seguidas por las solanas planas y convexas, y finalmente por las llanas más inaccesibles (GARCÍA-RUIZ et al., 1991).

El objetivo de este artículo es cuantificar los cambios que ha habido en la superficie de los usos del suelo, no sólo de los cultivos agrícolas, en un área modelo del Prepirineo catalán, y la influencia que han tenido la pendiente y la orientación en estos cambios mediante la comparación diacrónica de fotografías aéreas.

\section{2. Área de estudio}

El área de estudio es la cuenca de la Riera de Canalda, de 6577 ha, situada en los Prepirineos del Solsonés (Figura 1). Esta cuenca es la cabecera de la Ribera Salada, afluente del río Segre. La importancia de este río reside en el hecho de ser uno de los dos únicos cursos de agua permanente en la comarca del Solsonés y que vierte sus aguas en el pantano de Rialb, cuya puesta en funcionamiento está prevista para el año 2000.

Esta cuenca es representativa del Prepirineo. Está formada por conglomerados masivos y areniscas calizas en la cabecera, que derivan a estratos subhorizontales alternados de margas y areniscas en la parte inferior, característicos de la depresión central catalana. El relieve es tabular y frecuentan los afloramientos de conglomerados y areniscas en las partes altas y laderas. Los suelos son poco profundos, calizos y pedregosos. Predominan Ustorthents y Udorthents, típicos y líticos, en función del régimen de humedad y de la presencia o no de contacto lítico. El uso es fundamentalmente forestal, unas pendientes elevadas (raramente inferiores al $20 \%$ ) que dificultan cualquier implantación urbana o actividad agrícola o industrial. Los bosques son principalmente pinares (Pinus sylvestris y P. uncinata) (Figura 2), los cuales ocupan alrededor de la mitad del territorio. La superficie ocupada por matorrales y yermos es superior al $60 \%$. Los cultivos mayoritarios son forrajes: alfal- 


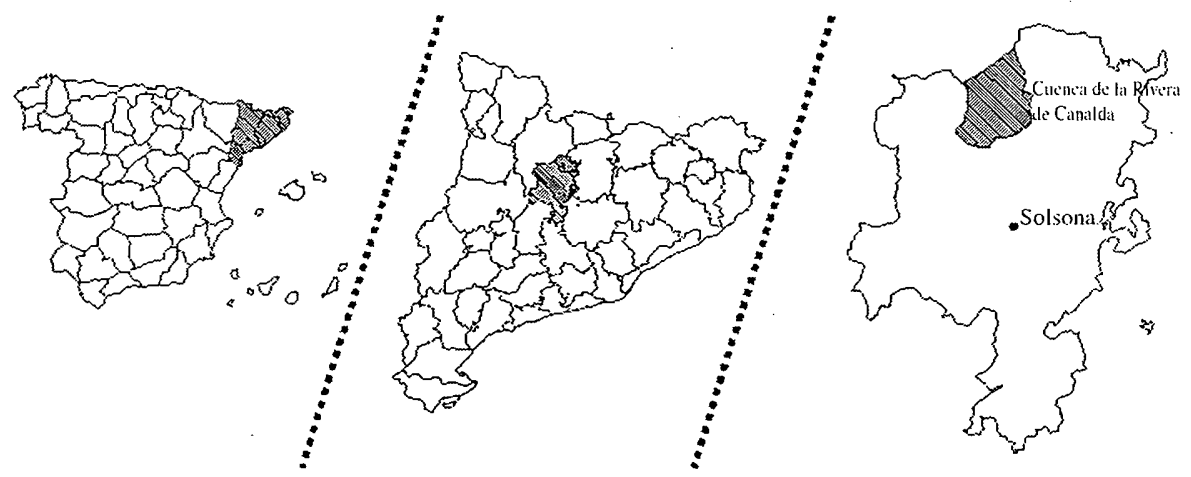

Figura 1. Situación de la zona de estudio. Location of the study area.

fa, esparceta y cereales. Localmente tiene importancia la patata de siembra en el municipio de Odèn.

La cuenca está orientada al sur (por lo que dominan las solanas) y presenta en conjunto un gran desnivel (altitudes comprendidas entre $660 \mathrm{~m}$ y 2383 $\mathrm{m})$, con predominio de altitudes superiores a los $1000 \mathrm{~m}$. Este desnivel produce un gradiente climático, y por ende de suelos y vegetación considerable: la precipitación anual varía de $600 \mathrm{~mm}$ en las partes bajas a $1200 \mathrm{~mm}$ en los pastos de alta montaña.

El gradiente altitudinal-climático en cuanto a los usos del suelo difiere del de otras cuencas de los Pirineos:

- Los pastos de alta montaña se encuentran por encima de los $1600 \mathrm{~m}$. Se caracterizan por el uso estacional. Este tipo de recursos se expandió por el hombre para aumentar el área de pasto, para facilitar un número mayor de cabezas de ganado y para alargar el período vegetativo

- En la base de la cuenca se encuentra el bosque y la mayor parte de los cultivos. El bosque se sitúa en las laderas y los cultivos en las pendientes más bajas, que coinciden con los estratos horizontales de areniscas calizas de la parte central de la cuenca. Los bosques han estado muy presionados en épocas de más población, principalmente los cercanos a explotaciones agrícolas

- En las partes más bajas de la cuenca se sitúa la mayor superficie de bosque, debido a que en esta zona no hay suficientes superficies llanas para poderse cultivar. Eso es debido a que la cuenca es de tipo torrencial, con la red de drenaje encajada, lo cual no permite el desarrollo de una llanura aluvial. 


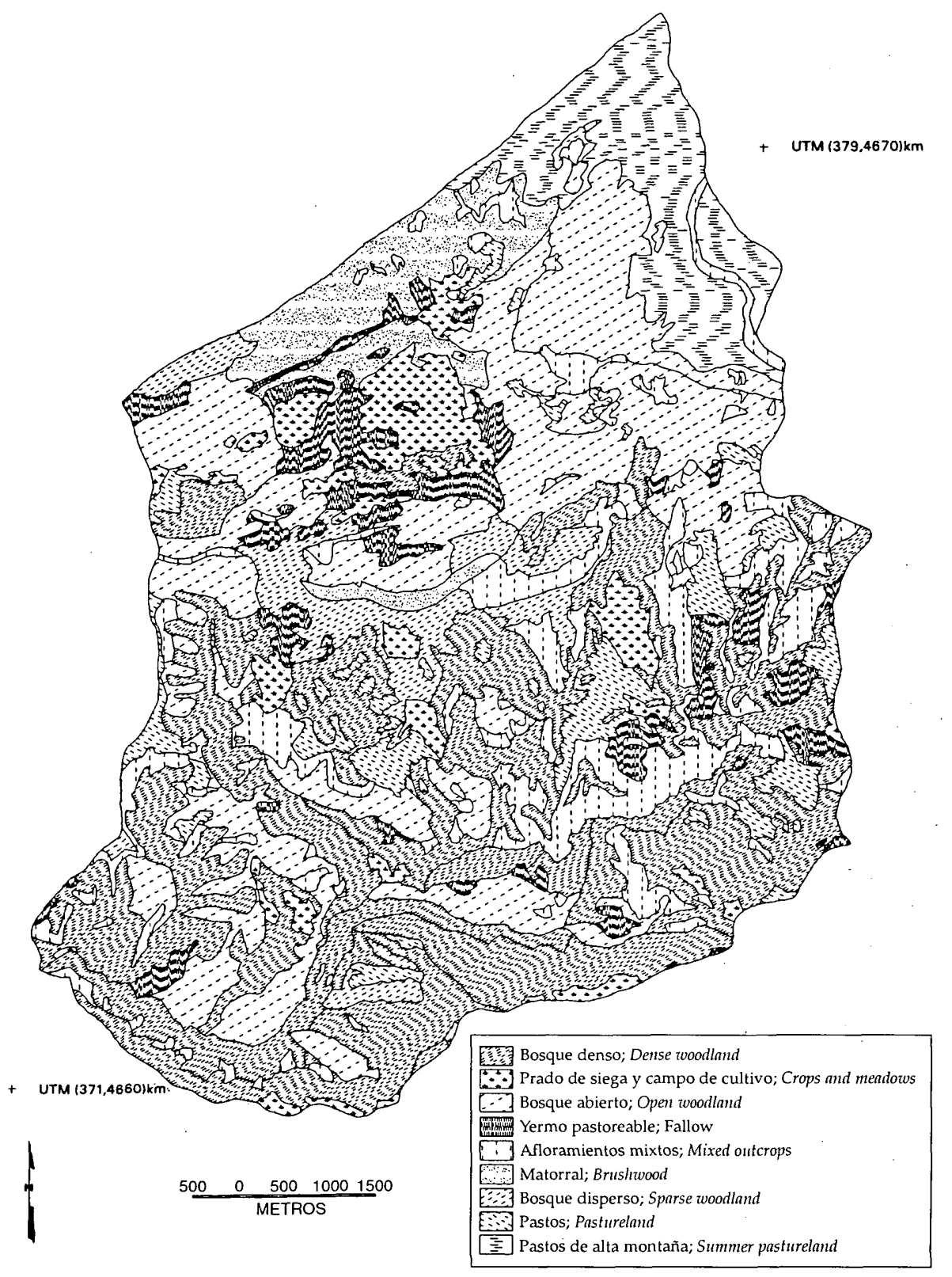

Figura 2. Mapa de usos del suelo del año 1993. Land use map, 1993. 
En el área de estudio ha habido un proceso de despoblación en el que se han abandonado gran número de explotaciones agrarias, lo cual ha dado lugar a un cambio en los usos del suelo. Al estudio de esos cambios dedicamos este trabajo.

La PAC (Política Agraria Comunitaria) ha tenido un impacto fuerte en esta zona (DPTOP-DGPAT, 1995) debido a la disminución pronunciada del precio de los cereales y también por el descenso de precios del vacuno de carne y consiguiente reducción de la carga ganadera. Este hecho no ha ayudado a la supervivencia de las explotaciones agrarias, que ya tienen suficientes dificultades por su situación en la montaña (aislamiento, dificultad de mecanización...).

\section{Material y métodos}

La metodología seguida se ha basado en la fotointerpretación secuenciada de fotografías aéreas de 1957 y 1992. También se han utilizado ortofotomapas del año 1993 o el mapa topográfico y se han hecho comprobaciones de campo. Las características de los documentos utilizados se recogen en la Tabla 1.

Para la elaboración de la leyenda de los usos del suelo, se ha estudiado previamente la adecuación a la realidad de diferentes clasificaciones utilizadas en trabajos previos. Los trabajos que se han consultado son, principalmente, el «Mapa de cultivos y aprovechamientos» del Ministerio de Agricultura, Pesca y Alimentación (escala 1/50.000, año 1989-1990), el Catastro de Rústica de los Ayuntamientos (años 1958 y 1996) y el «Censo Agrario" del Instituto Nacional de Estadística (diversos años).

Además de estos antecedentes, se han tenido en cuenta otra información como los usos que se pueden delimitar en las fotografías aéreas, la escala de trabajo, la homogeneidad de las clases de la leyenda y los objetivos del estudio. En la Tabla 2 incluimos la caracterización de los usos del suelo según su

\begin{tabular}{|lccc|}
\hline Material & Año & Escala & Tipo \\
\hline Fotografías aéreas & 1957 & $1 / 33.000$ (aprox.) & Blanco y negro \\
Fotografías aéreas & 1992 & $1 / 22.000$ (aprox.) & Blanco y negro \\
Ortofotomapas & 1993 & $1 / 25.000$ & Color \\
\hline
\end{tabular}

Tabla 1. Documentación básica. Basic documentation. 
aspecto en las fotografías aéreas. En la Figura 3 se han señalado varios usos en una fotografía aérea del año 1992.

\begin{tabular}{|c|c|c|}
\hline Uso & Característica determinante & Otras Caracteristicas \\
\hline Afloramientos & Color blanco puro & $\begin{array}{l}\text { Encontramos pocos con la } \\
\text { superficie mínima cartogra- } \\
\text { fiable. }\end{array}$ \\
\hline $\begin{array}{l}\text { Campos de cultivo } \\
\text { y prados de siega }\end{array}$ & Parcelas bien definidas & $\begin{array}{l}\text { Color más oscuro que los } \\
\text { afloramientos, pero muy } \\
\text { variable. Resulta difícil } \\
\text { distinguirlos. }\end{array}$ \\
\hline Pastos & Parcelas no definidas & $\begin{array}{l}\text { Formaciones herbáceas. Color } \\
\text { gris uniforme. }\end{array}$ \\
\hline Pastos de alta montaña & Color claro & $\begin{array}{l}\text { Formaciones herbáceas per } \\
\text { encima de los } 2000 \mathrm{~m} \text {. }\end{array}$ \\
\hline Yermos pastoreables & Color claro & $\begin{array}{l}\text { Se caracteriza por los árboles y } \\
\text { arbustos poco densos. } \\
\text { Alrededor de les explotaciones. }\end{array}$ \\
\hline Matorrales & $\begin{array}{l}\text { Puntos pequeños } \\
\text { y uniformes }\end{array}$ & $\begin{array}{l}\text { Formaciones arbustivas. } \\
\text { Color claro. }\end{array}$ \\
\hline Bosques & Color oscuro & $\begin{array}{l}\text { Buena estimación de la frac- } \\
\text { ción de cabida cubierta. }\end{array}$ \\
\hline
\end{tabular}

Tabla 2. Caracterización de los usos del suelo en las fotografías aéreas. Land use characterization in the aerial photographs.

La leyenda definitiva de usos del suelo que se ha utilizado, de acuerdo con la posibilidad de delimitar las diferentes unidades a escala $1 / 25.000$, y teniendo en cuenta los usos con un comportamiento hidrológico diferenciado, viene sintetizada en la Tabla 3.

Delimitando en las fotografías aéreas las diferentes unidades y utilizando los ortofotomapas como base cartográfica, se obtuvieron dos mapas a escala 1/25000 de los usos del suelo, uno del año 1957 (figura 4) y otro del año 1993 (Figura 2). Introduciendo estos mapas en un Sistema de Información Geográfica se obtuvo la superficie y la distribución de los diferentes usos. El SIG que se ha utilizado es el programa $\mathrm{ARC} / \mathrm{INFO}^{\circledR}$ (versión 7.04).

Mediante este SIG se ha podido encontrar los cambios de usos del suelo ocurridos entre los dos años de estudio, sobreponiendo los dos mapas. Además, se han establecido las tendencias de evolución de los usos según la pendiente y la orientación. Un paso previo ha sido la elaboración de los 


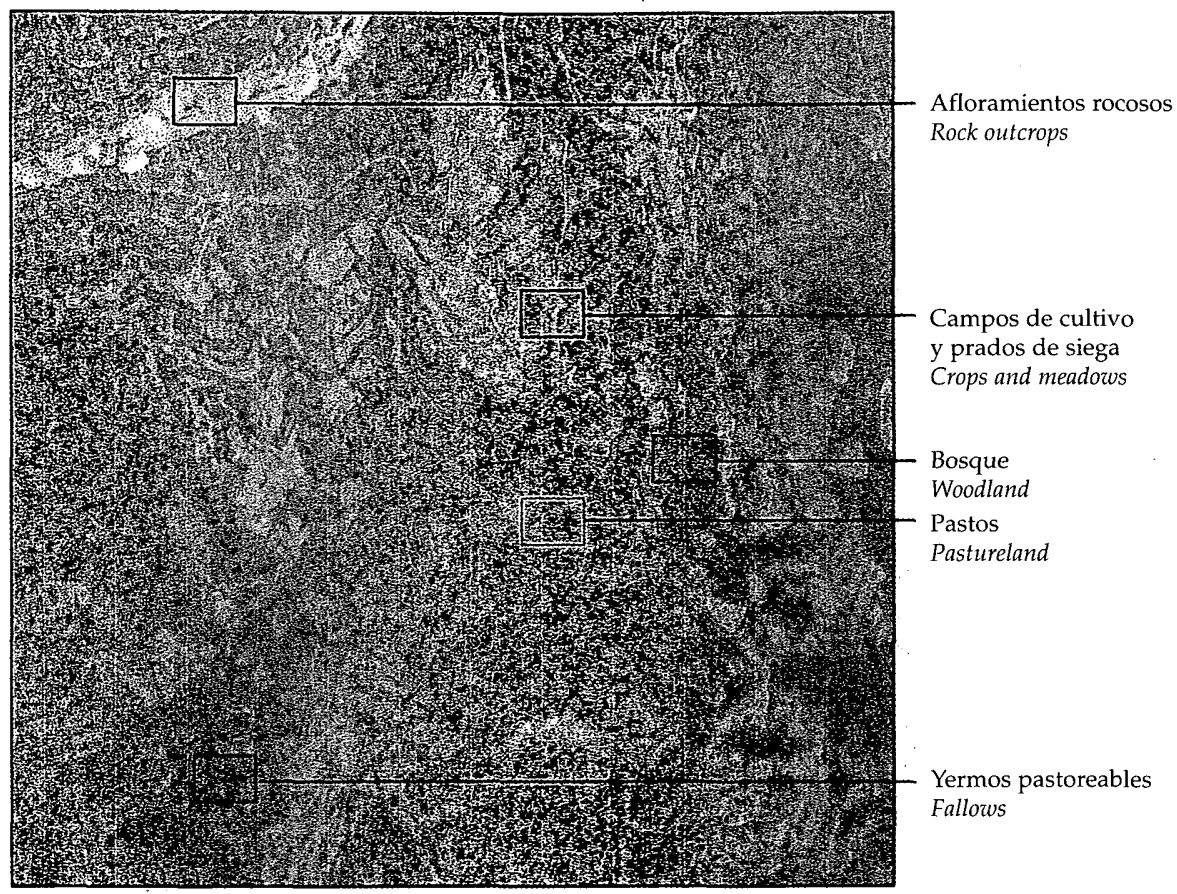

Figura 3. Aspecto de algunos usos del suelo en una fotografía aérea del año 1992 realizada por el Instituto Cartográfico de Cataluña.

Aerial photograph (Instituto Cartográfico de Cataluña,1992) showing the appearance of some of the surveyed land uses.

mapas de pendiente y orientación a partir del Modelo Digital del Terreno (MDT). La fuente del MDT es el mapa topográfico del «Institut Cartogràfic de Catalunya", a escala $1 / 50.000$ y equidistancia entre curvas de $20 \mathrm{~m}$.

También se han querido estudiar los cambios desde un punto de vista de su intensidad. Para eso se ha determinado la gradación de los usos del suelo de menor a mayor cobertura del mismo (Figura 5). Se han analizado los cambios que han tenido la misma magnitud de variación y en el mismo sentido, 
MONITORIZACIÓN DE LOS CAMBIOS DE USO DEL SUELO EN LA CABECERA DE CUENCA...

\begin{tabular}{|c|c|c|}
\hline Usos del suelo & Signo & Características \\
\hline Bosque denso & A & $\begin{array}{l}\text { Superficie cubierta por especies arbóreas con una } \\
\text { fracción de cabida cubierta (fcc) entre el } 70 \text { y el } \\
100 \% \text {. Su uso es el aprovechamiento forestal. }\end{array}$ \\
\hline Bosque abierto & $\mathrm{O}$ & $\begin{array}{l}\text { Superficie cubierta por especies arbóreas con una } \\
\text { fcc entre el } 40 \text { y } 70 \% \text {. Su aprovechamiento es el } \\
\text { forestal. }\end{array}$ \\
\hline Bosque disperso & $\mathrm{D}$ & $\begin{array}{l}\text { Superficie cubierta por especies arbóreas con una } \\
\text { fcc entre el } 10 \text { y el } 40 \% \text {. Su aprovechamiento es } \\
\text { forestal y eventualmente pastoral. }\end{array}$ \\
\hline Matorral & M & $\begin{array}{l}\text { Superficie cubierta por especies arbustivas con un } \\
\text { recubrimiento superior al } 10 \% \text { de la superficie. } \\
\text { Puede aprovecharse ocasionalmente por el ganado. }\end{array}$ \\
\hline Yermo pastoreable & E & $\begin{array}{l}\text { Superficie herbácea donde hay una colonización } \\
\text { arbórea y arbustiva, inferior al } 10 \% \text { de la superfi- } \\
\text { cie. Su uso es el pastoreo accidental de ganado } \\
\text { tipo ovino y caprino. }\end{array}$ \\
\hline Pasto & $\mathrm{P}$ & $\begin{array}{l}\text { Superficie herbácea que puede tener un cierto } \\
\text { recubrimiento arbóreo o arbustivo inferior al } 2 \% \text {. } \\
\text { El aprovechamiento principal es mediante la } \\
\text { acción directa de los rumiantes. }\end{array}$ \\
\hline Pasto de alta montaña & a PR & $\begin{array}{l}\text { Superficies herbáceas que no se agostan en el } \\
\text { periodo seco. Los encontramos a partir de los } \\
2000 \text { m de altitud. El aprovechamiénto es } \\
\text { mediante la acción directa de los rumiantes. }\end{array}$ \\
\hline $\begin{array}{l}\text { Prado de siega } \\
\text { y campo de cultivo }\end{array}$ & B & $\begin{array}{l}\text { Los cultivos son de tipo anual, principalmente } \\
\text { de cereal, planta forrajera o patata de siembra. } \\
\text { Los prados de siega son superficies herbáceas } \\
\text { donde el principal aprovechamiento es mediante } \\
\text { siega. También se puede pastorear. En la cuenca } \\
\text { sería de tipo natural, es decir, no se implanta nin- } \\
\text { guna mezcla de pratenses. Las únicas siembras } \\
\text { son de enriquecimiento cada cierto tiempo y } \\
\text { alguna labor para mejorar el prado. }\end{array}$ \\
\hline Afloramiento & $\mathrm{F}$ & $\begin{array}{l}\text { Son superficies sin vegetación donde la roca aflo- } \\
\text { ra. Son improductivas. }\end{array}$ \\
\hline Afloramiento mixto & $\mathrm{FM}$ & $\begin{array}{l}\text { Superficies donde entre los afloramientos hay } \\
\text { vegetación intercalada. Eśta vegetación no parece } \\
\text { haberse aprovechado en los últimos } 50 \text { años. }\end{array}$ \\
\hline
\end{tabular}

Tabla 3. Leyenda definitiva de los usos del suelo en la cuenca de la Riera de Canalda. Land use legend for the Riera de Canalda basin. 


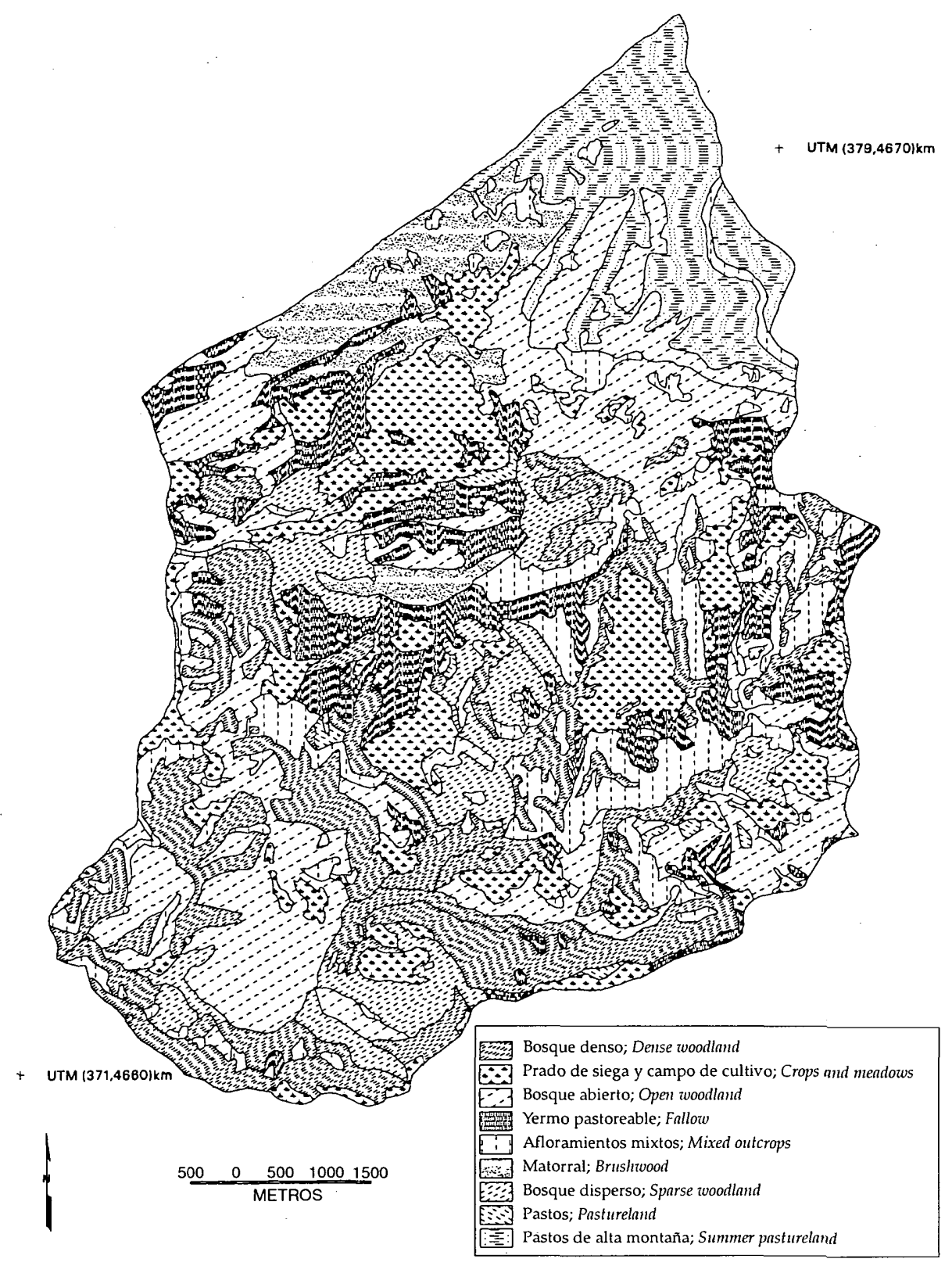

Figura 4. Mapa de usos del suelo del año 1957.

Land use map, 1957.

110 
es decir, que han saltado el mismo número de escalones de la gradación de la Figura 5 en el mismo sentido de las flechas.

Los criterios para establecer esta gradación son puramente morfológicos y responden a una protección creciente del suelo, debida a una mayor cobertura y complejidad de la cubierta. A pesar de ello se corresponden aproximadamente a los cambios secuenciales ordenados descritos por GÓMEZ-SAL et al. (1993) en campos abandonados la Cordillera Cantábrica.

Con este punto de vista, se ha querido analizar en qué pendientes y orientaciones se han dado dichos cambios. Esto responde a la importancia que tiene la cobertura del suelo en los procesos hidrológicos, debido a la intercepción y la disminución de la velocidad de la escorrentía. Por otro lado, no se tienen en cuenta aspectos del suelo, como la compactación, entonces este análisis no puede aplicarse para cuantificar los cambios hidrológicos de la cuenca. Un ejemplo es el paso de cultivo a pasto: se ha considerado que comporta una mayor protección del suelo, pero la posible compactación por el paso del ganado puede hacer que el pasto goce de una infiltración inferior a la del cultivo.

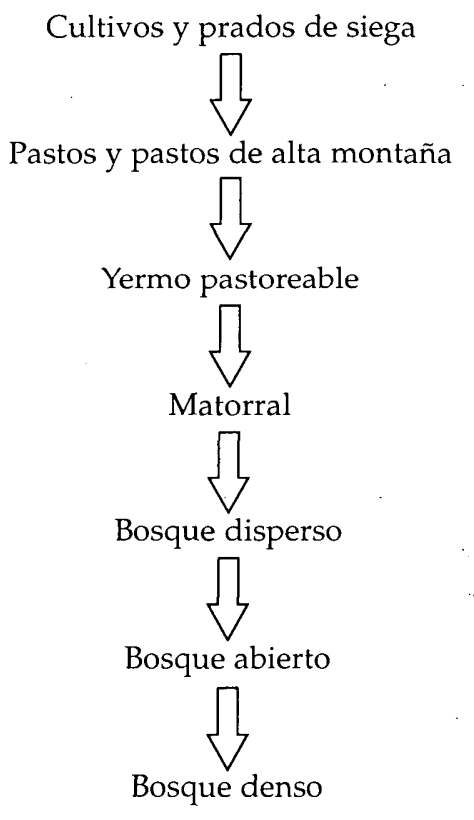

Figura 5. Evolución de los usos del suelo de menor a mayor protección del suelo. Land use evolution from less to more soil protection. 
PIRINEOS 153-154

\section{Resultados}

En la Tabla 4 se muestra la superficie que alcanzan los diferentes usos del suelo, tanto el año 1957 como actualmente, y en las Figuras 2 y 4 su distribución espacial. La Figura 8 muestra además los cambios de uso del suelo según la pendiente. Se observa que actualmente el uso del suelo predominante en la cuenca es el forestal. La superficie de este uso ya tenía proporciones elevadas el año 1957, un $49 \%$, cuando la presión humana era más elevada. Hoy en día, con la despoblación, ha aumentado su importancia tanto en superficie, un 11 $\%$, como en densidad: el bosque denso ha aumentado en 845 ha., que eran básicamente de bosque abierto y disperso. Eso se explica porque más de la mitad de la cuenca muestra más del $35 \%$ de pendiente, y en estas pendientes el uso más adecuado es el forestal, ya que se hace difícil la implantación de ningún cultivo o aprovechamiento agrícola. Un $64 \%$ del bosque se encuentra en estas pendientes.

El siguiente uso más importante era, el año 1957, los cultivos y los prados de siega, ocupando un $14 \%$ de la cuenca. Esta importancia ha disminuido drásticamente, siendo el uso que ha perdido más superficie: de 906 a 372 ha. Los cultivos y prados de siega se encuentran mayoritariamente en pendientes inferiores al $35 \%$. Se observa que donde más han desaparecido es en pendientes superiores al $35 \%$. El año 1957. se cultivaba un alto porcentaje, casi un $20 \%$ en un medio desfavorable, es decir, en laderas con una alta pendiente (superior al $35 \%$ ). En la mayoría de los casos estas laderas estaban abanca-

\begin{tabular}{|c|c|c|c|c|}
\hline & \multicolumn{2}{|c|}{ Usos año 1957} & \multicolumn{2}{|c|}{ Usos actuales } \\
\hline & $h a$ & $\%$ & ha & $\%$ \\
\hline Cultivos y prados de siega & 905,8 & 13,8 & 372,4 & 5,7 \\
\hline Pastos & 50,9 & 0,8 & 349,3 & 5,3 \\
\hline Pastos de alta montaña & 555,3 & 8,4 & 454,1 & 6,9 \\
\hline Yermos pastoreables & 750.0 & 11,4 & 394,5 & 6,0 \\
\hline Matorral & 365,7 & 5,6 & 350,8 & 5,3 \\
\hline Bosque disperso & 1493,4 & 22,7 & 1562,7 & 23,8 \\
\hline Bosque abierto & 516.0 & 7,8 & 307,9 & 4,7 \\
\hline Bosque denso & 1219,5 & 18,5 & 2064,8 & 31,4 \\
\hline Afloramientos & 110,4 & 1,7 & 110,4 & 1,7 \\
\hline Afloramientos mixtos & 609,9 & 9,3 & 609,9 & 9,3 \\
\hline TOTAL & 6577,0 & 100,0 & 6577,0 & 100,0 \\
\hline
\end{tabular}

Tabla 4. Superficie de los usos del suelo del año 1957 y los actuales, en la cabecera de la cuenca de la Ribera Salada. Surface distribution of the present and 1957 land uses on the Ribera Salada basin. 
ladas, y el tipo de uso agrícola evitaba los problemas de erosión (GARCÍA RUIZ et al., 1991). Eso probablemente era la causa de serios problemas de erosión. Actualmente, se cultiva un $13 \%$ en estas pendientes.

La principal función del área cultivada era garantizar un sistema de autosuficiencia en el que la alimentación básica necesaria estuviera asegurada. $\mathrm{Al}$ disminuir la presión humana y al entrar los Pirineos en un mercado más amplio y dinámico se tendió a intensificar el uso en los mejores suelos, mientras que en el área restante se extensificó (LASANTA \& RUIZ FLAÑO, 1990). Esta tendencia observada en todos los Pirineos, también se da en la cuenca: gran parte de los cultivos y prados se han abandonado dando lugar a superficies herbáceas, cuyo principal uso es el pastizal con aprovechamiento extensivo.

Superficie de los usos del suelo del año 1957 distribuida por intervalos de pendiente (en \%)

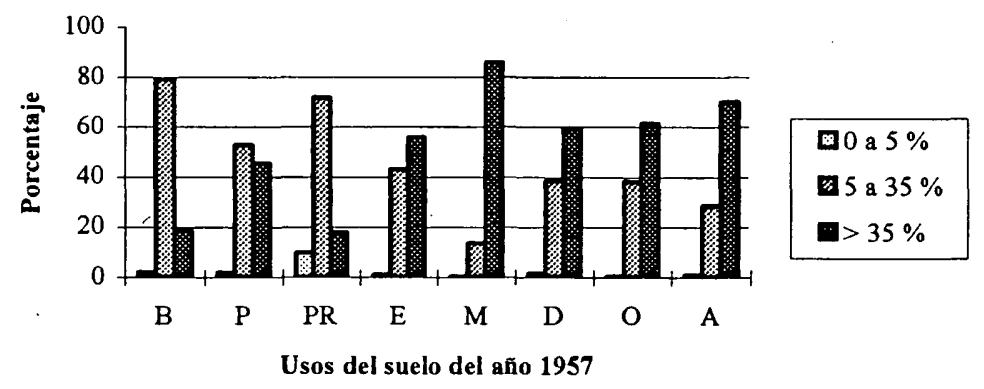

Superficie de los usos del suelo actuales distribuida por intervalos de pendiente (en \%)

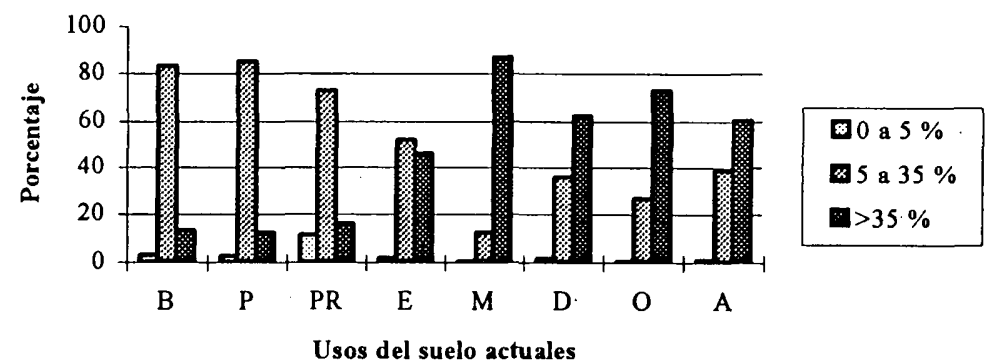

Figura 8. Distribución de la superficie de los usos del suelo del año 1957 y actuales según la pendiente. B: cultivos y prados de siega, P: pastos, PR: pastos de alta montaña, E: yermos pastoreables, M: matorral, D: bosque disperso, $\mathrm{O}$ : bosque abierto, A: bosque denso.

Surface distribution of the present and 1957 land uses according to slope. 
De esta manera, los pastos han sido el uso que ha aumentado más debido al abandono de cultivos y prados, pasando de 51 a 349 ha. A pesar de eso, los pastos tienen poco peso dentro de la cuenca, ocupando un $5 \%$ de superficie. Los pastos están frecuentemente sometidos a procesos erosivos, favorecidos por la compactación provocada por el paso del ganado y agravados en las pendientes elevadas.

Por otro lado, los pastos de alta montaña y los yermos pastoreables han disminuido debido a la colonización arbórea, ocupando los pastos de alta montaña un $7 \%$ y los yermos un $6 \%$. El peso de estos usos no era, aún así, muy elevado el año 1957: un $8 \%$ los pastos de alta montaña y un $11 \%$ los yermos. Este también es un efecto directo de la disminución de la intensidad de la actividad humana en los Pirineos. Una menor presión de pastoreo ha favorecido la instalación de vegetación arbórea.

Las superficies que se han mantenido invariables han sido los afloramientos rocosos, que ocupan un $10 \%$ de la cuenca. En los afloramientos con vegetación intercalada no ha habido ningún aprovechamiento debido a la dificultad del terreno. Se ve la importancia de los afloramientos en toda la cuenca, ya que superan todos los usos menos el matorral y el bosque.

Para el conjunto de la cuenca, la superficie que ha variado de uso ha sido de 2313 ha (un $35 \%$ ). Atendiendo a la evolución de los usos expuesta en la Figura 5, la mayoría de estos cambios han sido positivos en cuanto a la protección del suelo, es decir, han seguido el sentido de la flecha. De las 2313 ha, 2045 corresponden a progresivos y 267 a regresivos. Debido a las limitaciones del método no se puede saber si ha habido una progresión continua, pues no hay datos de épocas intermedias. Para poder interpretar los resultados, se han agrupado los cambios de dos maneras:

Según la intensidad del cambio, es decir, se han agrupado los cambios que han saltado el mismo número de escalones de la evolución de los usos del suelo.

Según el uso actual, es decir, se han reunido todos los cambios que han evolucionado a un mismo uso final.

Las superficies de los cambios según estos dos enfoques están reflejadas en la Tabla 5 y 6 y en los mapas de cambios (Figuras 6 y 7). Las principales observaciones que se pueden hacer son las siguientes:

- Los cambios regresivos más intensos son los de menor superficie. Eso demuestra la baja tendencia que hay a intensificar los usos, tal como se ha visto en todos los Pirineos

- Los cambios progresivos más importantes son de cuatro niveles, que se corresponden con el paso de cultivos, prados, pastos y yermos a superficies arboladas

- El cambio progresivo más importante es el cambio a bosque denso, que se corresponde a lo que se observa en la Tabla 6 
MONITORIZACIÓN DE LOS CAMBIOS DE USO DEL SUELO EN LA CABECERA DE CUENCA...

\begin{tabular}{|ccc|}
\hline Niveles de cambio & Progresivos & Regresivos \\
\hline 1 & 930.73 & 189.68 \\
2 & 514.98 & 32.91 \\
3 & 130.03 & 21.95 \\
4 & 320.71 & 12.56 \\
5 & 78.75 & 8.68 \\
6 & 70.21 & 1.67 \\
\hline
\end{tabular}

Tabla 5. Superficie (en ha) de los cambios según el grado de intensidad. Ha of every land use change following the intensity.

\begin{tabular}{|lcc|}
\hline Cambio a: & Progresivos & Regresivos \\
\hline Bosque denso & 916.36 & - \\
Bosque abierto & 450.02 & 140.38 \\
Bosque disperso & 268.1 & 33.03 \\
Matorral & 5.7 & 0.52 \\
Yermo pastoreable & 119.75 & 30.05 \\
Pasto de alta montaña & 0 & 26.43 \\
Pasto & 285.48 & 25.88 \\
Cultivos y prados & - & 11.18 \\
\hline
\end{tabular}

Tabla 6. Superficie (en ha) de los cambios según el uso final. Ha of every land use change following the final use.

- El cambio regresivo más importante es el cambio de bosque denso a abierto (140.38 ha). Este cambio está localizado en una zona y se correspondería un aprovechamiento forestal.

El paso siguiente ha sido establecer las tendencias de estos cambios según la pendiente y la orientación. Para eso se ha hecho un análisis similar al realizado con los usos del suelo, determinando que porcentaje de superficie de cada tipo de cambio estaba dentro de cada intervalo de pendiente y de orientación. No todos los resultados obtenidos han permitido establecer unas tendencias de evolución: se ha visto que la pendiente es una variable más relacionada con los cambios de uso que la orientación.

Las principales tendencias que se han establecido en el análisis de los cambios según su intensidad son las siguientes:

- El cambio regresivo más intenso (cambio de bosque a cultivos y prados) es el que tiene más proporción en zonas llanas. Por otro lado, los menos intensos tienen más superficie en pendientes elevadas (Figura 9)

- Las zonas llanas son las que tienen menos cambios progresivos. La mayoría de estos cambios se dan en pendientes medias. 


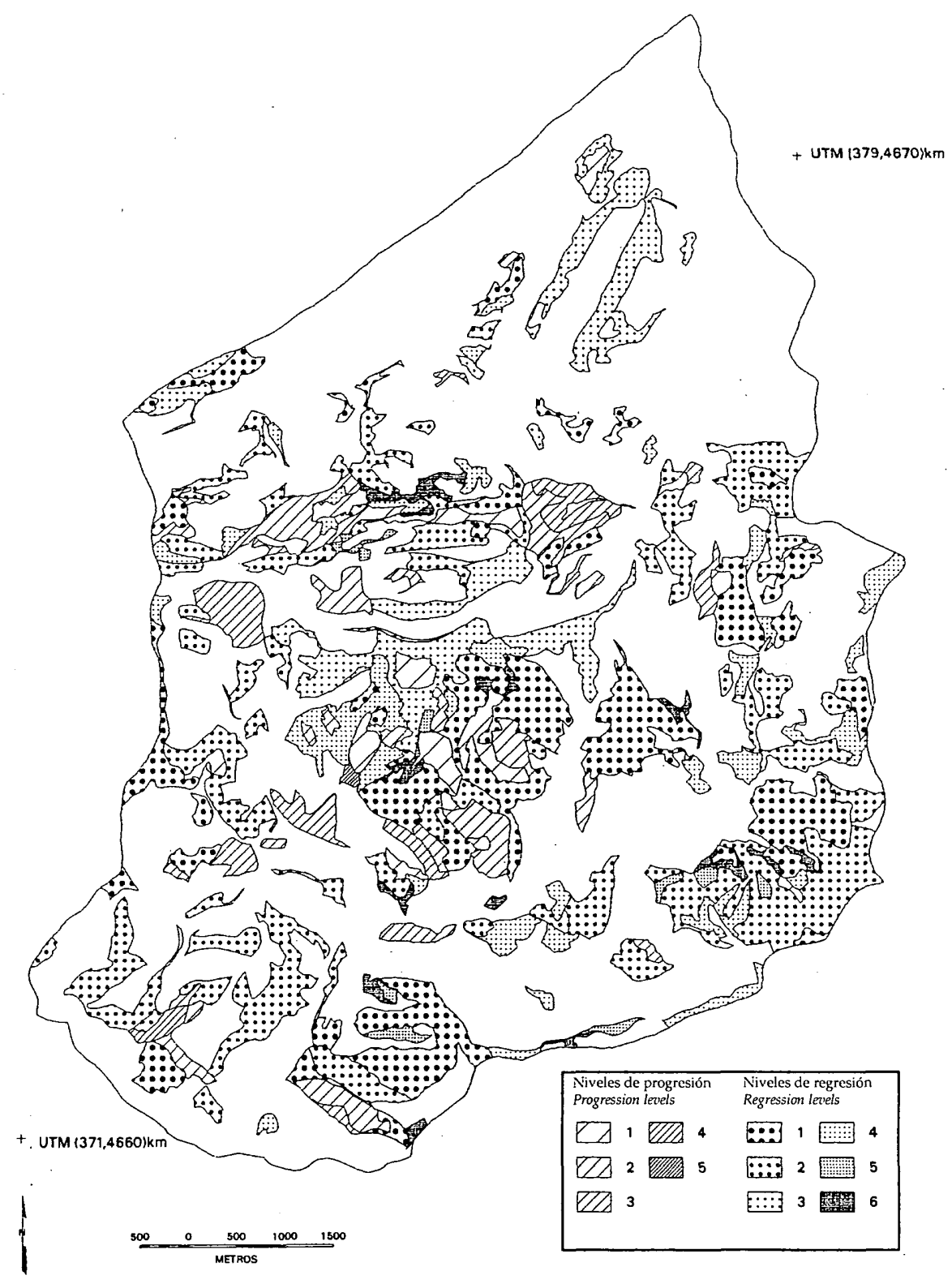

Figura 6. Mapa de intensidades de cambio. Change intensity map.

116 
MONITORIZACIÓN DE LOS CAMBIOS DE USO DEL SUELO EN LA CABECERA DE CUENCA..

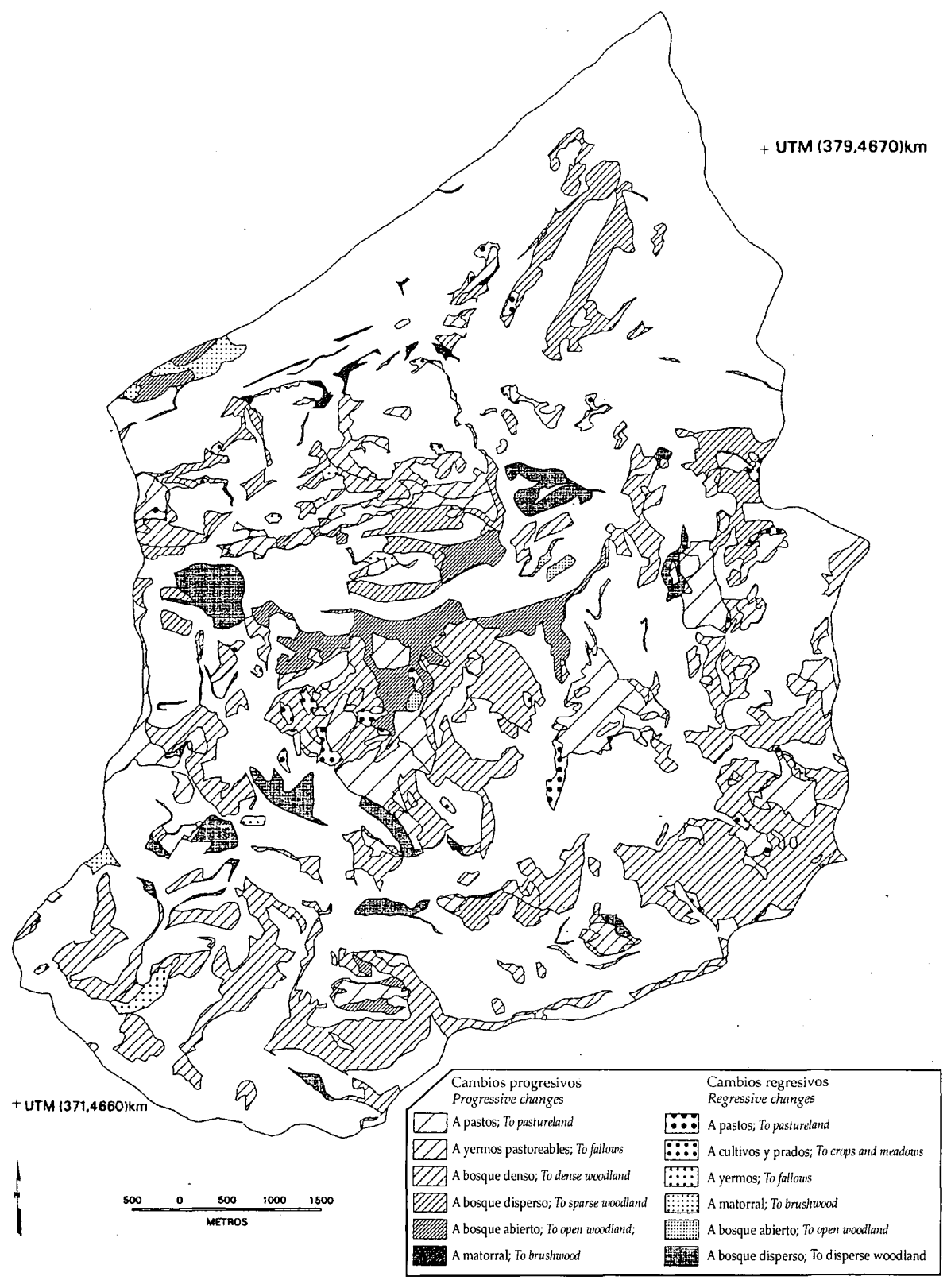

Figura 7. Mapa de cambios según el uso final. Map of changes according to final land use. 


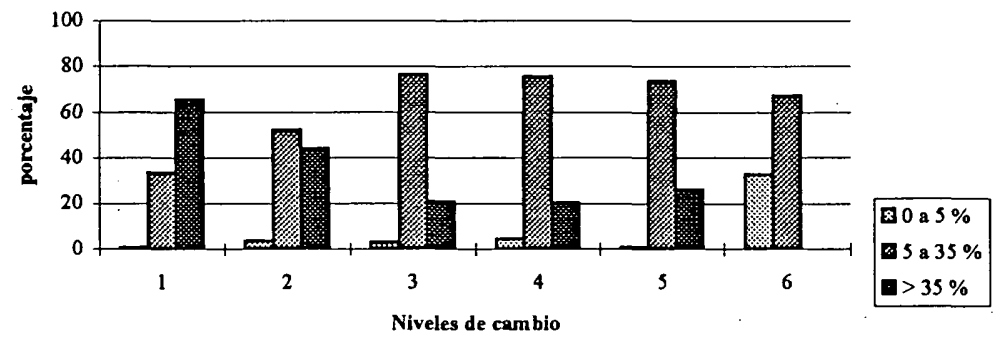

Figura 9. Porcentaje de superficie de los cambios regresivos según la intensidad en cada intervalo de pendiente. En el eje de las abscisas se indica el número de pasos de la gradación de la Figura 5 que comprende el cambio.

Surface percentage of regressive changes according to change intensity in each slope interval.

El cambio progresivo más intenso (de cultivos y prados a bosque) es el que tiene más componente norte. Respecto a los cambios regresivos no se ve ninguna relación con la orientación (Tabla 7).

Las principales tendencias en el análisis de los cambios según el uso actual son:

- La mayor parte del paso de cultivos y prados a pasto se da en pendientes medias. Eso responde a la tendencia de continuar cultivando zonas llanas y abandonar las pendientes medias. En los otros cambios progre-

\begin{tabular}{|c|c|c|c|c|c|c|c|}
\hline \multicolumn{8}{|c|}{ Cambios progresivos } \\
\hline $\begin{array}{l}\text { Uso finall } \\
\text { Orientación }\end{array}$ & $\begin{array}{l}\text { Bosque } \\
\text { denso }\end{array}$ & $\begin{array}{l}\text { Bosque } \\
\text { abierto }\end{array}$ & $\begin{array}{l}\text { Bosque } \\
\text { disperso }\end{array}$ & Matorral & $\begin{array}{c}\text { Yermo } \\
\text { pastoreable }\end{array}$ & Pastos & $\begin{array}{l}\text { Pastos de } \\
\text { alta montaña }\end{array}$ \\
\hline Norte & 22.4 & 9.2 & 13.0 & 0.0 & 16.4 & 11.7 & 0.0 \\
\hline Este & 26.9 & 18.9 & 10.2 & 13.7 & 6.7 & 15.0 & 0.0 \\
\hline Sur & 21.5 & 43.7 & 53.4 & 86.2 & 27.7 & 44.4 & 0.0 \\
\hline Oeste & 29.2 & 28.2 & 23.4 & 0.1 & 49.1 & 28.9 & 0.0 \\
\hline \multicolumn{8}{|c|}{ Cambios regresivos } \\
\hline $\begin{array}{l}\text { Uso finall } \\
\text { Orientación }\end{array}$ & $\begin{array}{c}\text { Cultivos y } \\
\text { prados de siega }\end{array}$ & Pastos & $\begin{array}{l}\text { Pastos de } \\
\text { alta montaña }\end{array}$ & $\begin{array}{c}\text { Yermo } \\
\text { pastoreable }\end{array}$ & Matorral & $\begin{array}{l}\text { Bosque } \\
\text { disperso }\end{array}$ & $\begin{array}{l}\text { Bosque } \\
\text { abierto }\end{array}$ \\
\hline Norte & 10.1 & 6.4 & 4.4 & 13.5 & 0.0 & 14.1 & 8.8 \\
\hline Este & 12.6 & 5.8 & 21.8 & 5.4 & 0.0 & 5.9 & 21.0 \\
\hline Sur & 28.5 & 51.1 & 47.9 & 50.4 & 3.2 & 79.8 & 27.2 \\
\hline Oeste & 47.8 & 36.7 & 26.0 & 30.7 & 96.7 & 0.2 & 43.0 \\
\hline
\end{tabular}

Tabla 7. Cambios de uso del suelo según el uso final en función de la orientación de la ladera (\% de superficie).

Land use changes following the final use and the slope exposure, (surface \%). 
sivos hay más superficie en pendientes altas, no mucha más que en pendientes medias, excepto en el paso a yermo pastoreable (Figura 10)

- Si bien los cambios regresivos tienen importancia en pendientes medias, en las pendientes altas tiene un peso importante el paso hacia usos que representan menor cobertura del suelo. En el otro extremo hay que remarcar el $15 \%$ de la superficie de zonas llanas que han pasado a cultivos y prados (Figura 11)

- En el análisis de los cambios, tanto progresivos como regresivos, según la orientación, no se establece ninguna tendencia. Lo que se ve es el dominio de la orientación sur y oeste en los cambios, con el predominio general de la primera (Tabla 7).

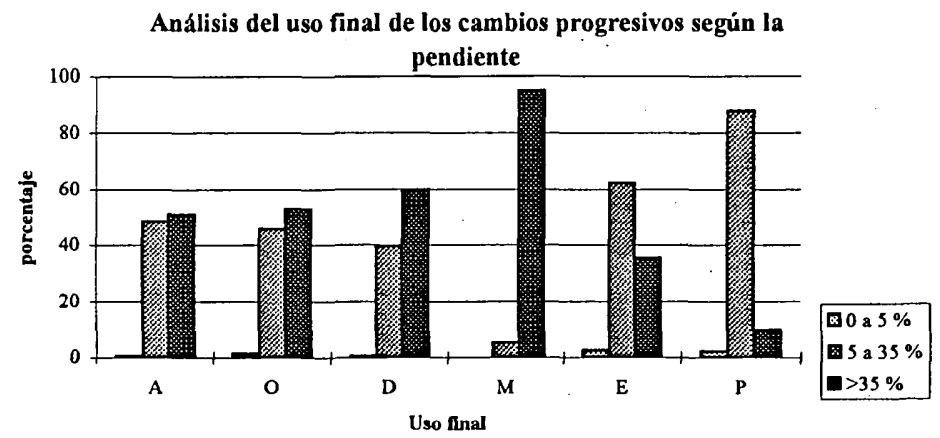

Figura 10. Porcentaje de superficie de los cambios progresivos según el uso final en cada intervalo de pendiente

A: bosque denso, O: bosque abierto, D: bosque disperso,

M: matorral, E: yermos pastoreables, P: pastos.

Surface percentage of progressive changes according to final land use in each slope interval.

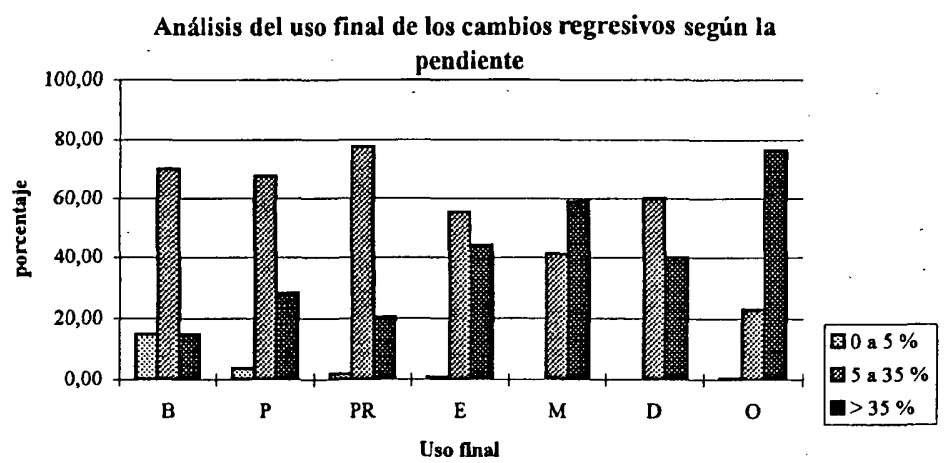

Figura 11. Porcentaje de superficie de los cambios regresivos según el uso final en cada intervalo de pendiente. B: cultivos y prados de siega, P: pastos, PR: pastos de alta montaña,

E: yermos pastoreables, M: matorral, D: bosque disperso, $\mathrm{O}$ : bosque abierto.

Surface percentage of regressive changes according to final land use in each slope interval. 


\section{Conclusiones}

La disminución de la población de los núcleos de la cuenca, y del Prepirineo en general, desde el año 1950 hasta la actualidad ha causado unos cambios en los usos del suelo. Los bosques, que son el uso predominante en la cuenca, han aumentado su superficie y en algunos casos su densidad. En la zona estudiada este incremento se debe a la disminución de la superficie de matorral, yermos y pastos de alta montaña. Los pastos, a pesar de ocupar poco en el total de la cuenca, son los que más aumentaron su superficie debido al abandono de cultivos y prados de siega, que han disminuido mucho su área.

Estos cambios han seguido unas pautas según diferentes variables. En este trabajo se han encontrado tendencias de cambios según la pendiente y la orientación. Estas dos variables del terreno ya se han relacionado con los cambios de usos del suelo en otros estudios (GARG \& HARRISON, 1992 ).

Los cambios progresivos se han dado la mayoría en pendientes medias y altas. Las zonas llanas casi no han cambiado, manteniéndose los cultivos y prados y los pastos de alta montaña de la parte más septentrional de la cuenca. Los cambios regresivos aumentan su intensidad al disminuir la pendiente. Los nuevos campos de cultivo y prados ocupan un porcentaje importante en zonas llanas.

La mayoría de los cambios progresivos y regresivos se dan en orientaciones sur y oeste. Los cultivos y prados que se han abandonado y estaban en orientación norte tienen más tendencia a ser actualmente bosques.

Los cambios acaecidos se han analizado teniendo en cuenta los diferentes grados de recubrimiento de los usos del suelo desde dos enfoques. Por un lado, se vio el grado de variación del recubrimiento del suelo y en qué sentido se ha dado (análisis según la intensidad del cambio). Por otro lado, se han cuantificado los recubrimientos que se han conseguido actualmente (análisis según el uso final). El primer enfoque permite localizar las áreas que han variado más desde el punto de vista de la hidrología del suelo y el segundo permite localizar los recubrimientos que influyen en el comportamiento hidrológico actual de la cuenca.

Estos enfoques han permitido mostrar que ha habido cambios de uso que repercuten necesariamente en la hidrología de la cuenca, basándose en el recubrimiento del suelo. No obstante, se necesita analizar muchos otros componentes, como por ejemplo la compactación del suelo, para poder llegar a resultados cuantitativos sobre la variación de la hidrología de la cuenca.

En conclusión, conviene impulsar estudios hidrológicos en cuencas de montaña, debido a la importancia que tiene el conocimiento del impacto del abandono del mundo rural en los caudales líquidos y sólidos, que a menudo 
van a parar a infraestructuras de gran envergadura, como es el pantano de Rialb en el río Segre, del cual la Ribera Salada conforma la mayor subcuenca por su margen izquierda.

Agradecimientos. Estudio realizado en el marco del Programa de Formación de Investigadores en "Dinámica Hidrológica y Manejo de Cuencas Forestales en Areas de Montaña Mediterránea», a su vez dentro del programa operativo 940503 ES5-Eje prioritario de Recursos Humanos-F.4.4 del Fondo Social Europeo (Centre Tecnològic Forestal de Catalunya, Solsona).

\section{Referencias}

BORDIU BARREDA, E. (1985). Valoración de la infrautilización en la Sierra de Ayllón y aportación de un modelo alternativo. Anales de la Universidad Complutense (Madrid), 5: 167-187.

BOSQUE MAUREL, J. (1968). Tradición y modernidad en las Alpujarras granadinas. Aportación española al XXI Congreso Geográfico Internacional, Madrid: 164-183,.

CABERO DIEGUEZ, V. (1980). Espacio agrario y economía de subsidencia en las montañas galaico-leonesas. Institución "Fray Bernardino de Sahagún» (C.S.I.C.), León y Ediciones de la Universidad de Salamanca, 134 pp.

DEBUSSCHE, M.; RAMBAL, S. \& LEPART, J. (1987). Land-use changes in the wet Mediterranean region: assessment of hydrological effects. Acta Oecologica, Oecologia Applicata, 8: 317-332.

DPTOP-DGPAT (Departament de Política Territorial i Obres Públiques. Direcció General de Planificació i Acció Territorial) (1995). Pla Comarcal de Muntanya 1995-1999. El Solsonès. Generalitat de Catalunya, Barcelonà. 232 pp.

GARCÍA-RUIZ, J. M.; LASANTA, T. \& SOBRÓN, I. (1985). Estudio comparado de la evolución geomorfológica de campos abandonados y áreas repobladas de la cuenca del Jubera. Comunidad Autónoma de la Rioja, Logroño. Informe, $345 \mathrm{pp}$.

GARCÍA-RUIZ, J. M. (1988). La evolución de la agricultura de montaña y sus efectos sobre la dinámica del paisaje. Revista de Estudios Agrosociales, 146: 5-37.

GARCÍA-RUIZ, J. M., RUIZ-FLAÑO, P., LASANTA, T., MONTSERRAT, G., MARTÍNEZ-RICA, J. P. \& PARDINI, G. (1991). Erosion in abandoned fields, what is the problem?. En SALA, M., RUBIO, J. L. \& GARCÍA-RUIZ, J. M. (eds.). Soil erosion studies in Spain: 97-108. Geoforma Ediciones, Logroño. 
GARG, P. K. \& HARRISON, A. R. (1992). Land Degradation and Erosion Risk Analysis in S.E. Spain: A Geographic Information System Approach. Catena, 19: 411-425.

GÓMEZ-SAL, A., ÁLVAREZ, J., MUÑOZ-YANGUAS, M. A. \& REBOLLO, S. (1993). Patterns of change in the agrarian landscape in an area of the Cantabrian Mountains (Spain) - Assessment by transition probabilities. En BUNCE, R. G. H., RYSZKOWSKI L. \& PAOLETTI, M. G. (eds.): Landscape ecoloy and agrosystems: 141-152. Lewis.

LASANTA, T. (1988). The process of desertion of cultivated areas in the Central Spanish Pyrenees. Pirineos, 132: 15-36.

LASANTA, T. (1989). Evolución reciente de la agricultura de montaña: modelos en El Pirineo Aragonés. Geoforma Ediciones. Logroño.

LASANTA, T. \& RUIZ-FLAÑO, P. (1990). Especialización productiva y desarticulación espacial en la gestión reciente del territorio en las montañas de Europa occidental. En GARCÍA-RUIZ, J. M. (ed.): Geoecología de las áreas de montaña: 267-295. Geoforma Ediciones, Logroño.

ORTEGA VALCÁRCEL, J. (1974). La transformación de un espacio rural. Las montañas de Burgos. Servicio de Publicaciones de la Universidad de Valladolid, $531 \mathrm{pp}$. 